\title{
Modificación de las propiedades ópticas y mecánicas de polímeros mediante implantación iónica
}

\author{
R.J. RODRÍGUEZ ${ }^{(1)}$, A. PÉrez (2), J. A. GARCíA (1), BLAS GARRIDO (2) J. MORANTE (2) \\ (1) Centro de Ingeniería Avanzada de Superficies AIN, Cordovilla-Pamplona
}

(2) Departament d’Electrònica-Facultat de Física, Martí i Franquès, 1, 08028 Barcelona

\begin{abstract}
En el presente trabajo se estudian los efectos introducidos por la implantación de Nitrógeno atómico y Silicio sobre probetas de policarbonato empleadas para usos ópticos. Distintas dosis de Nitrógeno y Silicio fueron implantadas de cara a poner de manifiesto el efecto de la dosis sobre las propiedades ópticas y mecánicas. Se llevaron a cabo ensayos mecánicos de microdureza, nanodureza, y AFM, así como ensayos ópticos de Reflexión-absorción IR y Transmitancia UV-VIS. Los resultados muestran un endurecimiento superficial para las implantaciones a dosis altas de Nitrógeno, así como cambios considerables en los espectros de transmitancia.

Palabras clave: Implantación, polímeros, óptica, dureza.
\end{abstract}

\section{Modification of optical and tribological properties of polymers by ion implantation}

This paper gathers the studies of the effects introduced when Nitrogen and Silicon were implanted in policarbonates used in optical applications. Different Nitrogen and Silicon doses were implanted for finding out the roles of this parameter in the mechanical and optical modifications. Several mechanical tests were carried out, like Microindentation, Nanoindentation and AFM. IR and UV-VIS optical tests were also carried out. Results show harder surfaces for the high Nitrogen implanted doses, and great changes in the UV-VIS spectrums.

Keywords: Implantation, polymer, optical, hardness.

\section{INTRODUCCIÓN}

La implantación iónica, como técnica de modificación superficial, esta siendo cada vez más utilizada para mejorar las características de los polímeros empleados en aplicaciones mecánicas, médicas, ópticas y eléctricas (1-3). En los últimos años se han realizado numerosos estudios sobre modificación de las propiedades de polímeros por implantación iónica ( 4-8). En estos trabajos se atribuyen los cambios en las propiedades del material al efecto ionizador del bombardeo iónico. En estas circunstancias el bombardeo con iones ligeros $(\mathrm{H}+$, $\mathrm{He}+\mathrm{o} \mathrm{N}+$ ) favorece la disipación de energía por ionización, y la formación de enlaces cruzados entre las cadenas poliméricas, produciéndose un endurecimiento superficial. En el caso de la implantación de iones pesados la mayor parte de la energía es transferida por desplazamiento de núcleos, produciéndose en este caso un ablandamiento superficial. Cuando se implanta Nitrógeno también juega un papel importante su incorporación al material (9).

\section{PROCEDIMIENTO EXPERIMENTAL}

\subsection{Materiales}

Las muestras consisten en discos plano-paralelos de $65 \mathrm{~mm}$ de diámetro de policarbonato.

\subsection{Tratamientos y caracterización.}

Las implantaciones se realizaron con un implantador de alta corriente del Centro de ingeniería de Avanzada de
TABLA I: RESUMEN DE LAS IMPLANTACIONES REALIZADAS.

\begin{tabular}{|lccc|}
\hline Substrato & Especie & Dosis & Energía \\
\hline Policarbonato & $\mathrm{N}^{+}$ & $5 \mathrm{e} 14 \mathrm{át} / \mathrm{cm}^{2}$ & $150 \mathrm{KeV}$ \\
Policarbonato & $\mathrm{N}^{+}$ & $1 \mathrm{e} 15 \mathrm{át} / \mathrm{cm}^{2}$ & $150 \mathrm{KeV}$ \\
Policarbonato & $\mathrm{N}^{+}$ & $5 \mathrm{e} 15 \mathrm{át} / \mathrm{cm}^{2}$ & $150 \mathrm{KeV}$ \\
Policarbonato & $\mathrm{N}^{+}$ & $1 \mathrm{e} 16 \mathrm{át} / \mathrm{cm}^{2}$ & $150 \mathrm{KeV}$ \\
Policarbonato & $\mathrm{Si}^{+}$ & $5 \mathrm{e} 14 \mathrm{á} / \mathrm{cm}^{2}$ & $100 \mathrm{KeV}$ \\
Policarbonato & $\mathrm{Si}^{+}$ & $1 \mathrm{e} 15 \mathrm{át} / \mathrm{cm}^{2}$ & $100 \mathrm{KeV}$ \\
\hline
\end{tabular}

Superficies de AIN. Los parámetros más relevantes de las implantaciones se muestran en la Tabla I.

La caracterización superficial de las muestras se ha realizado en primer lugar a partir de un microscopio óptico que permite determinar la corrugación de la superficie en el rango micrométrico. En segundo lugar, se ha empleado un AFM nanoscope para el estudio de la corrugación a escala nanométrica, que permite la obtención de imágenes con resolución atómica. La dureza Universal H.U. de los materiales tratados y $\sin$ tratar se ha medido a cargas de $2 \mathrm{mN}, 5 \mathrm{mN}$ y $25 \mathrm{mN}$, empleando un Microdurómetro FICHERSCOPE H100, con capacidad para realizar ensayos con cargas por debajo de 1 $\mathrm{mN}$.

Por último también se han realizado espectros de transmisión en el rango visible y ultravioleta empleando un espectroscopio dispersivo Shimadzu UV-VIS 2101 PC. 


\section{RESULTADOS}

\subsection{Caracterización AFM}

\subsubsection{IMPLANTACIÓN DE NITRÓGENO.}

La caracterización AFM para las probetas implantadas con $\mathrm{N}^{+}$ mostró en primer lugar un efecto de suavizado o pulido de las ralladuras propias de la fabricación. En la figura 1 puede observarse que las probetas sin implantar y las de baja dosis (arriba), presentan ralladuras del pulido, mientras que las probetas con implantaciones a dosis media (5 e15 át $/ \mathrm{cm}^{2}$ ) y alta (1 e16 át/ $\mathrm{cm}^{2}$ ), representadas en la parte inferior, presentan una superficie lisa.

En lo que se refiere al umbral de presión de rotura, puede observarse en la Figura 2 que la probeta implantada con 5e14 át/cm2 se "rompe" con menos de $100 \mathrm{GPa}$, mientras que las probetas con dosis de 5e15 y 1 e16 át/cm2 no se "rompe" para cargas muy superiores.

\subsubsection{IMPLANTACIÓN DE SILICIO}

Las pruebas de nanoindentación realizadas con el Microscopio de Fuerzas Atómicas sobre las probetas implantadas con Si mostraron un umbral de presión para la rotura de unos $200 \mathrm{Gpa}$ en comparación con los menos de 100 Gpa de las muestras sin implantar. Esto confirma el aumento de la resistencia a la abrasión a nivel nanométrico.

\subsection{Ensayos de Microdureza}

De cara a estudiar la modificación de la superficie implantada se llevaron a cabo ensayos de microdureza a baja carga. Como puede observarse en la Figura 3, donde se muestran las curvas de carga y descarga para las probetas sin implantar y la implantada con Nitrógeno a dosis de 5 e15 át/cm2 y 1 e16 át $/ \mathrm{cm} 2$, la implantación de estas dosis de Nitrógeno produce un aumento considerable de la dureza superficial. En esta figura, y en las Figura 4 y 5, donde se muestran la dureza frente a la profundidad, y la dureza diferencial frente a la profundidad, puede también observarse la rotura de la capa modificada por la implantación en el cambio de pendiente a aproximadamente 0.4 micras de penetración del indentador. Estos resultados están corroborados por ensayos de nanoindentación donde se observa el mismo efecto de rotura ( Figura 6).

Por otro lado cabe destacar que la "rotura" de la capa se produce aproximadamente en el pico de máxima concentración de la implantación, como queda reflejado en la Figura 6. En esta figura se muestra el perfil de la implantación de $1 \mathrm{e} 16$ át $/ \mathrm{cm} 2$ de Nitrógeno, realizada con el programa de simulación TRIM.

\subsection{Espectros de Transmitancia}

En la Figura 8 se muestran los espectro de transmitancia para las probetas de referencia y para las distintas dosis de Nitrógeno implantado. En esta figura puede observarse como disminuye la transmitancia para las probetas implantada. Esta disminución en la transmitancia es mayor en la zona verde-azúl del espectro, llegando hasta valores por debajo del $20 \%$ para las implantaciones a altas dosis.

En la Figura 9 se muestra el espectro de transmitancia para las implantaciones de Si. En esta gráfica puede observarse una disminución de hasta un $60 \%$ en la transmitancia para la implantación a dosis de 1e15 át/ cm2 en la zona verde-azúl del espectro.

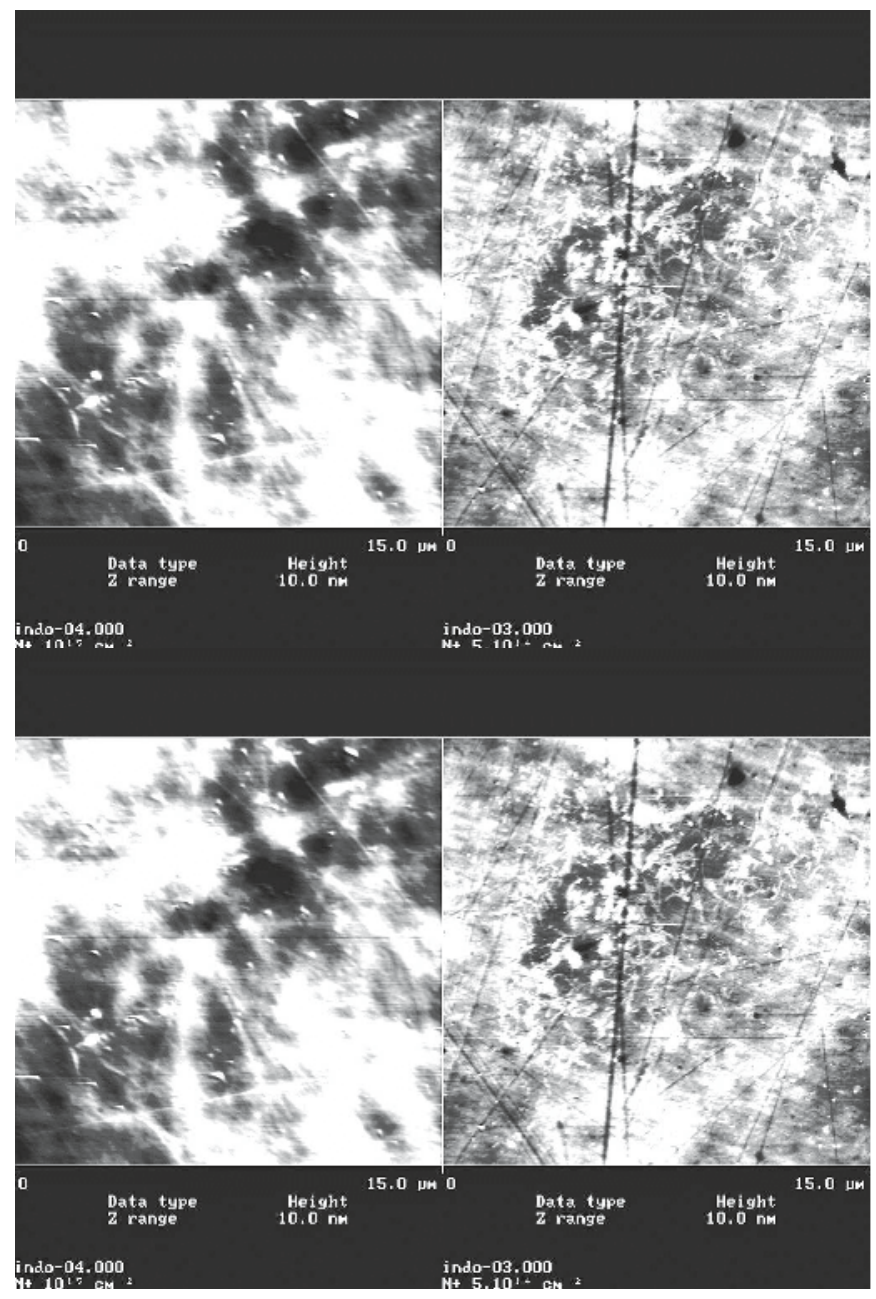

Figura 1: AFM para las probetas implantadas con Nitrógeno. Arriba las dosis bajas, debajo las dosis altas.

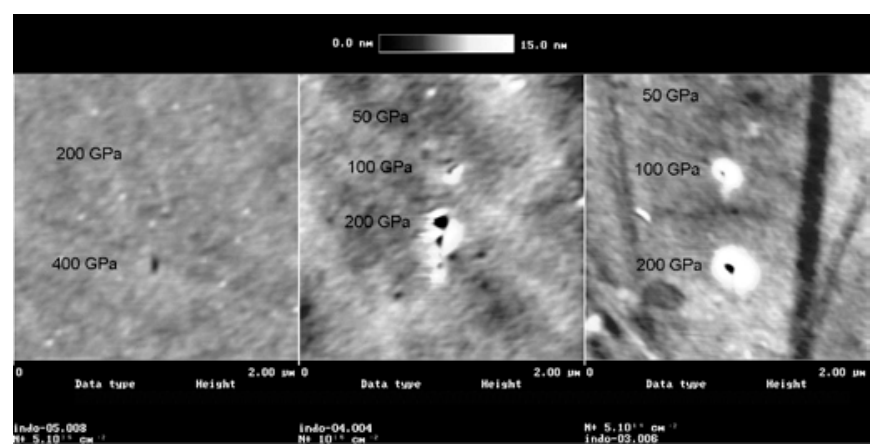

Figura 2: AFM de las probetas implantadas con Nitrógeno con dosis (de izquierda a derecha) 5e15,1e15 y 5e14. 


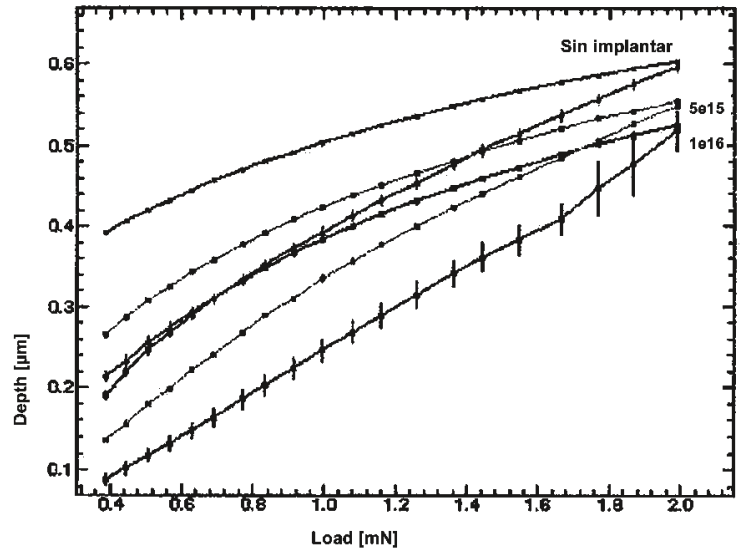

Figura 3: Curvas de carga-descarga para las probetas implantadas con Nitrógeno a dosis altas y sin implantar.

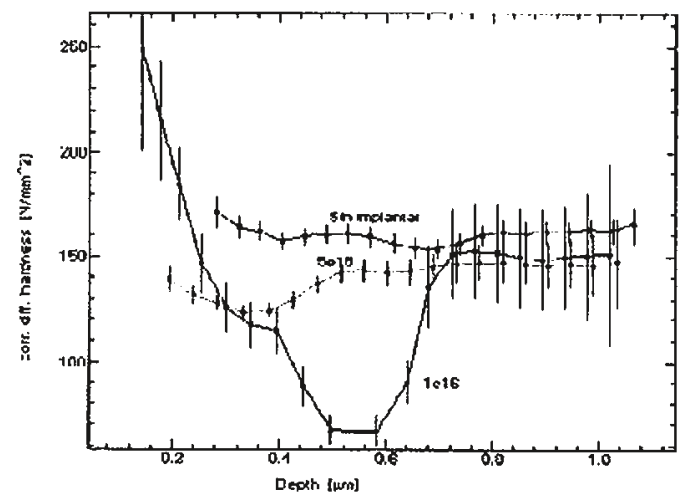

Figura 5: Curvas de dureza diferencial para las dosis altas de Nitrógeno y para la probeta sin implantar.

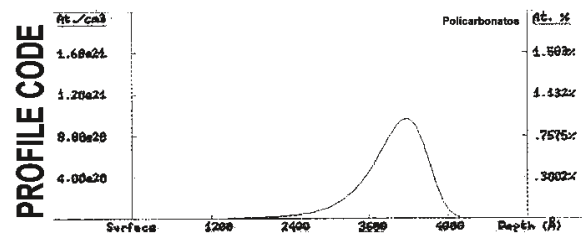

Figura 7: Simulación del perfil de concentración para la implantación de $1 \mathrm{e} 16$ át. $/ \mathrm{cm}^{2}$ de Nitrógeno en Policarbonato.

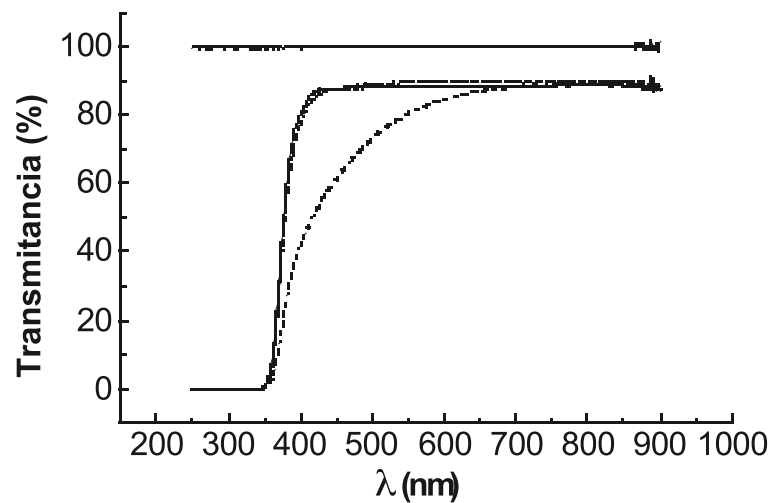

Figura 9: Curvas de transmitancia para las probetas implantadas con $\mathrm{Si}$ (punteadas) y la de referencia.

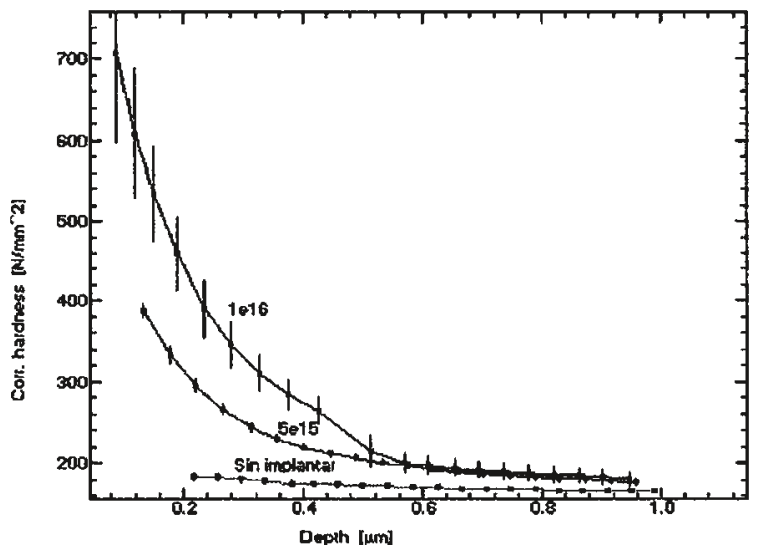

Figura 4: Curvas de dureza a $2 \mathrm{mN}$ de carga final para las probetas implantadas con dosis altas de Nitrógeno y sin implantar

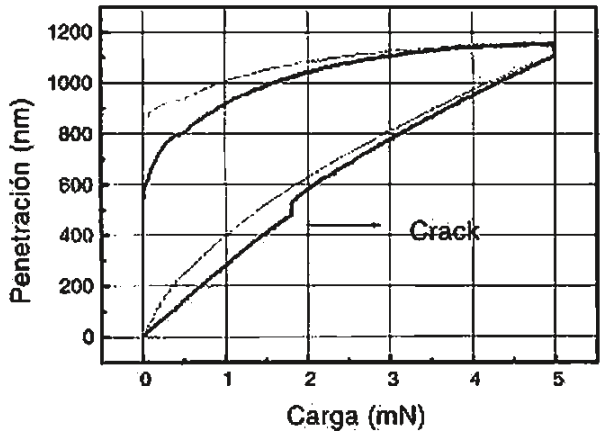

Figura 6: Curva de nanoindentación para la probeta sin implantar(gris) y la probeta con dosis $1 \mathrm{e} 16$ át $/ \mathrm{cm}^{2}$ (negro).

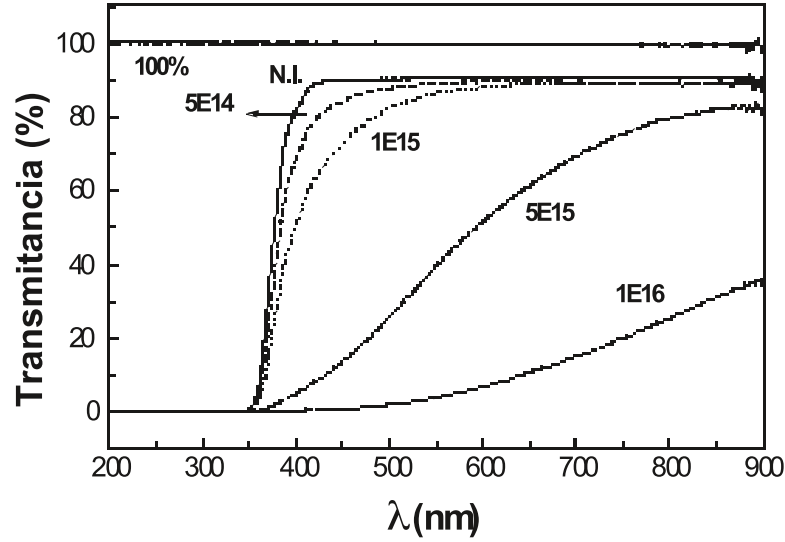

Figura 8: Curvas de transmitancia para las probetas implantadas con Nitrogeno (punteadas)y para la sin implantar. 


\section{CONCLUSIONES}

La implantación de altas dosis de Nitrógeno atómico (>5e15át./ cm2), producen aumentos de hasta un $60 \%$ en la dureza superficial del Policarbonato. Estos aumentos de dureza son mayores para las dosis más altas de Nitrógeno atómico.

La implantación de Nitrógeno atómico sobre policarbonato a dosis mayores de 5e15át./ $\mathrm{cm} 2$ produce superficies libres de las ralladuras del pulido.

La transmitancia para las implantaciones de nitrógeno se ve alterada para las dosis mayores de 1e15át./cm2, en mayor medida para las dosis más altas, y preferentemente en la zona verde-azul del espectro.

Los resultados de las implantaciones de Silicio no revelan cambios en las propiedades mecánicas a escala micrométrica (Microdurómetro), pero si a escala nanométrica. Las muestras implantadas con dosis de 1e15 mostraron un aumento del umbral de presión de rotura de 100 GPa a 200 Gpa.

La transmitancia para las implantaciones de Silicio no se ve alterada considerablemente para las dosis bajas, pero si en las dosis altas en las que se degrada hasta un $25 \%$ en la zona verde-azul del espectro.

\section{BIBLIOGRAFÍA}

1. J.C. Pivin, P. Colombo, Ceramic coatings by ion irradiation of polycarbosilanes and polysiloxanes J. Mater. Sci. 32 6175-6182 (1997).

2. Rung Ywan Tsai, et al. "Properties of optical thin films and coatings prepared by reactive electron-beam deposition with and without ion bombardments". Opt. Eng. 36 (12) 3433-3438 (1997)

3. D. M. Rück, J. Schulz, N. Deusch. "Ion irradiation on polymeric surfaces". Nucl. Instr. And Meth. B 131 149-158 (1997).

4. B. Pignataro, M.E. Fragalà, O. Puglisi. "Crosslinking of polysilanes by ion beam irradiation". Nucl. Instr. And Meth, B 131 141-148 (1997).

5. L. Zhang et. al. "Effects of ion bombardment on polymer films". Mater. Chem. And Phys. 54 98-101 (1998).

6. B. J. Briscoe, L. Fiori, E. Pellillo, "Nano-indentation of polymeric surfaces". J. Phys. D: Appl. Phys. 31 2398-2404 (1998).

7. H. Dong, W. Shi, T. Bell. "State of the art overview: ion beam surface modification of polymers towards improving tribological properties". Surf. and Coat. Thech. 111 29-40 (1999).

8. H. Dong, T. Bell. "Potential of improving tribological performance of UHMWPE by engineering the Ti6Al14V counterfaces". Wear 8021 (1998) $\mathrm{xxx}$

9. A. Tóth, T. Bell, Z. Bertoiti, M. Mohai,B. Zelei. "Surface modification of polyethylene low KeV ion beams". Nucl. Instr. And Meth. B000(1998)000.000. 